
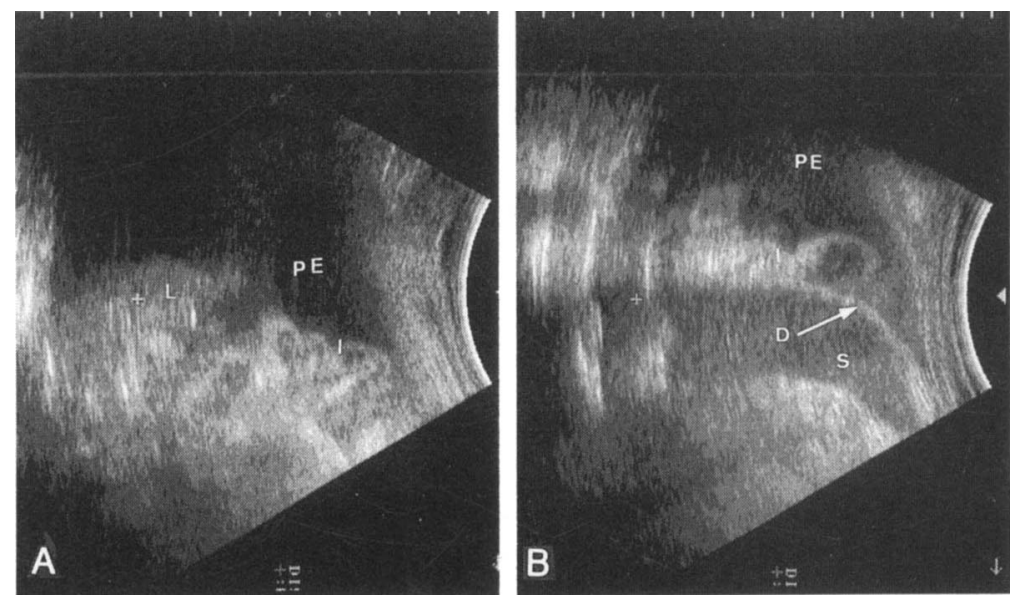

Fig. 2. A and B, Ultrasonic examination of the chest revealed loops of the small bowel in the left pleural cavity and a left-sided pleural effusion with pulmonary compression. Note the typical ultrasonic finding of a transverse section of the small bowel (B). $S$, Spleen; $D$, diaphagm; $L$, compressed lung; $P E$, pleural effusion; $I$, loops of intestine.

and angiographic midterm results in 200 patients. $\mathrm{J}$ THORAC CARdiovasc SuRg 1993;105:615-23.

\section{Transdiaphragmatic herniation of the stomach after right gastroepiploic artery grafting}

\section{To the Editor:}

In recent years, the right gastroepiploic artery (RGEA) has been used as a second reliable arterial graft for coronary artery bypass grafting $(\mathrm{CABG})$, along with the internal thoracic artery (ITA). ${ }^{1}$ Few abdominal postoperative complications after RGEA grafting have been reported, such as pancreas-derived hyperamylasemia or pancreatitis, ${ }^{2}$ hemoperitoneum caused by bleeding of the RGEA pedicle, ${ }^{2}$ perforated duodenal ulcer, ${ }^{3}$ and incisional ventral hernia. ${ }^{1}$ Recently, two of our patients who had received an $R G E A$ graft required surgical treatment because of transdiaphragmatic gastric herniation along the RGEA pedicle, a complication never before reported to our knowledge.

The first patient, a 46-year-old man, underwent a double CABG in February 1993, 3 months after an anterior myocardial infarction. The left ITA was anastomosed sequentially to the second diagonal branch and the left anterior descending coronary artery. The RGEA was routed posterior to the stomach and the left lobe of the liver, brought through a $1.5 \mathrm{~cm}$ long slit in the posterior part of the diaphragm (posterior route), and anastomosed to a posterolateral branch of the right coronary artery. The patient was discharged on the ninth postoperative day with aspirin and $\beta$-blocker therapy. Between the third and ninth postoperative weeks he was treated successfully with oral corticosteroids for postpericardiotomy syndrome. Eight months later, he was readmitted in a unit for general surgery because of compulsive vomiting, hiccups, and acute pain in the left upper quadrant of the abdomen. Palpation of this region caused great pain but revealed no masses, and peristalsis was within normal limits. Blood pressure was $160 / 100 \mathrm{~mm} \mathrm{Hg}$, and he had no fever. ST-segment depression was present in leads $D 3$ and $a V_{F}$ of the electrocardiogram. A chest X-ray film showed a paracardial air shadow on the left side and compression of the lower lobe of the left lung (Fig. 1). Roentgenograms demonstrated normal passage of swallowed contrast medium (Gastrografin) through the esophagus to the cardia and filling of a paracardial mass by a narrow transdiaphragmatic channel (Fig. 2). Urgent laparotomy confirmed a strangulation of almost the entire stomach because of thoracic herniation through the diaphragmatic orifice for the RGEA pedicle, which showed severe congestion and cyanosis. After enlargement of the diaphragmatic orifice and reduction of the gastric herniation, the perfusion of the involved organs was rapidly restored and the RGEA was still pulsatile. A gastropexy was performed to prevent recurrences. Postoperatively, the ischemic changes on the electrocardiogram disappeared completely, and no cardiac enzyme elevation was noted. The patient was discharged on the tenth day.

The second patient, a 45-year-old man, underwent a quadruple CABG in April 1992: left ITA graft to the left anterior descending artery, right ITA graft to a marginal branch, saphenous vein graft to a posterolateral branch of the circumflex artery, and RGEA graft to the posterior descending branch of the right coronary artery via the posterior route. He left the hospital on the ninth day with a regimen of $\beta$-blocker and aspirin therapy. The man remained free of symptoms until October 1993, when nausea and epigastric pain radiating to the left shoulder suddenly developed. Clinical examination showed no abnormalities except for epigastric tenderness, without signs of an acute abdominal problem. Arteriography confirmed patency of the RGEA graft. Barium x-ray examination and gastroscopy disclosed a large "hiatal" hernia and several ulcerations in the herniated portion of the stomach. During laparoscopic exploration, a herniation of the fundus of the stomach was found and easily reduced, and the diaphragmatic orifice was narrowed 


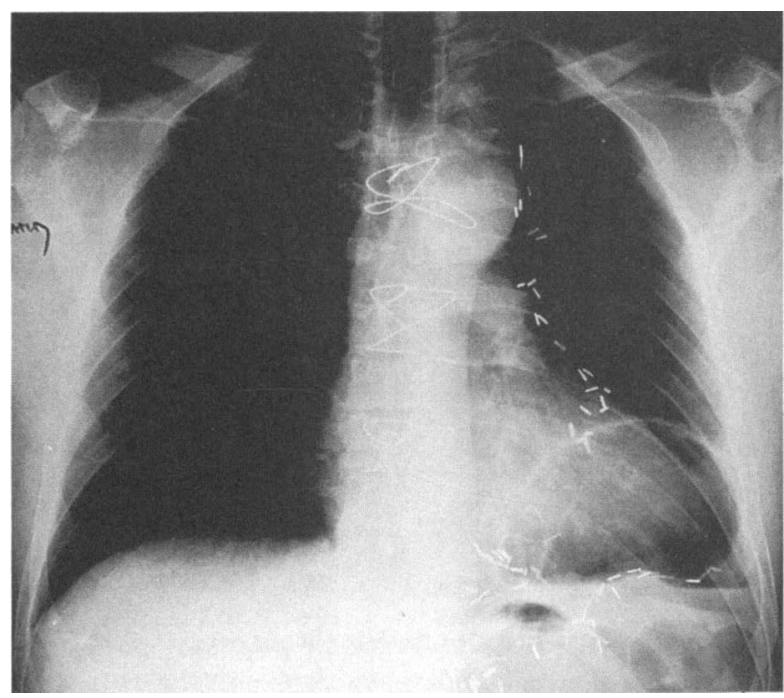

Fig. 1. Chest $x$-ray film showing paracardial air shadow with compression of left lower lung.

by one 2-0 Ethibond suture (Ethicon, Inc., Somerville, N.J.). The patient was discharged on the fourth day after an uneventful postoperative course.

The possibility of a diaphragmatic hernia through the orifice for the RGEA pedicle should be considered whenever a patient with this specific graft has acute abdominal symptoms such as pain, vomiting, hiccups, or a combination of these symptoms. Symptoms of esophagitis are absent, because this type of hernia causes no gastroesophageal reflux. The electrocardiogram might show ischemic changes in the territory supplied by the RGEA graft. A large hernia might be suspected on plain chest $\mathrm{x}$-ray films by the presence of an air shadow with sometimes an air-fluid level in the lower left part of the chest. The localization of the stomach with regard to the diaphragm might be helped by the position of the radiopaque clips placed along the gastric greater curvature during preparation of the RGEA graft. However, the ultimate diagnosis should be made by water-soluble contrast $\mathrm{x}$-ray studies or gastroscopy. The danger not only of gastric complications such as strangulation, ulceration, bleeding, or even perforation, but also of impairment of the RGEA graft with myocardial ischemia or even infarction, indicate surgical treatment. This includes reduction of the hernia, appropriate narrowing of the diaphragmatic orifice, and sometimes gastropexia to prevent recurrences. This therapy seems possible by a laparoscopic approach in selected cases, as illustrated by our second patient. Gastric ulcerations should be managed by adjuvant medical treatment.

Several factors can theoretically favor the occurrence of a hernia through the diaphragmatic orifice for the RGEA graft. One is an excessive orifice having been made too large at the first operation or having widened afterward. Second, the close apposition of the stomach and the diaphragmatic orifice of a retrohepatically routed RGEA graft predisposes theoretically to gastric herniation, in contrast to the anterior route, in which the liver protects against possible gastric herniation. Factors leading to elevated intraabdominal pressure, such as ascites, obesity, chronic cough, pregnancy, or blunt thoracoabdominal trauma, could not be found in our patients. ${ }^{4}$ Tissue weakening by long-term corticosteroid therapy in the first patient might have played a role. To prevent this complication, we now preferen-

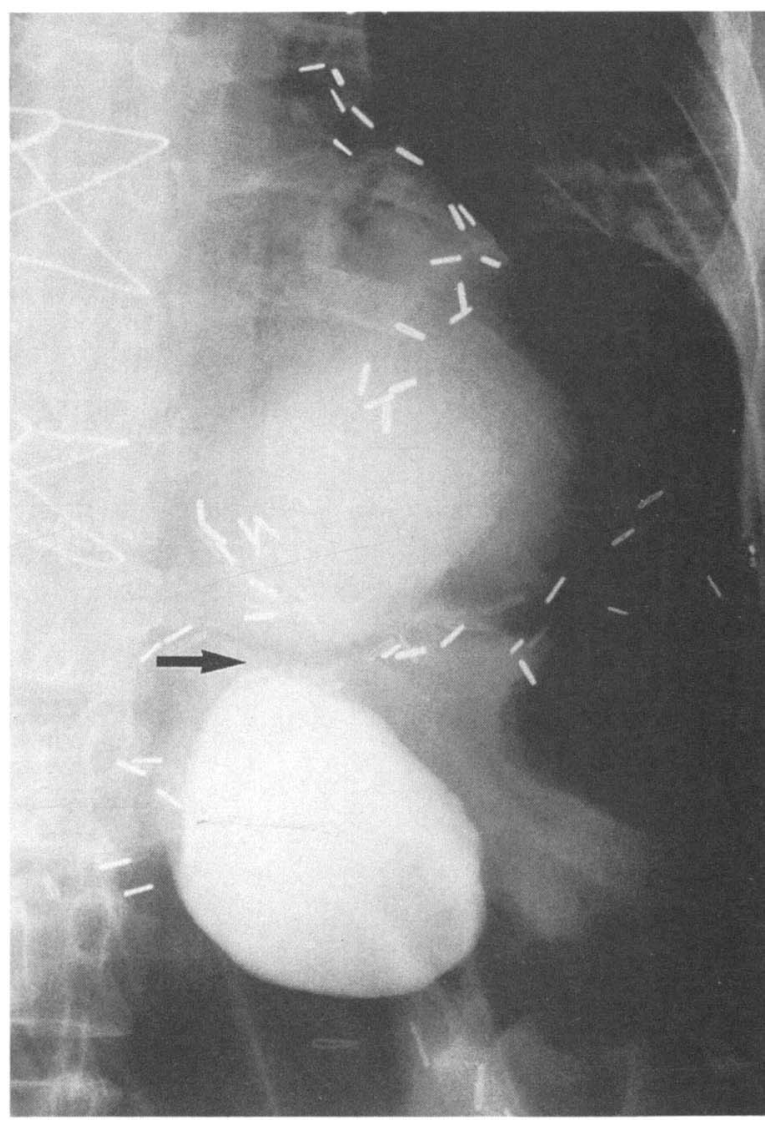

Fig. 2. Roentgenogram after contrast medium (Gastrografin) swallow showing narrow channel (arrow) filling diaphragmatic hernia

tially use the anterior route, which is also technically easier and is favored by many authors, ${ }^{1,3}$ except in rare cases in which the RGEA graft is too short to reach the posterior side of the heart. Moreover, we take care to make the orifice for the RGEA graft not too large and to create it in the central fibrous part instead of the lateral, more muscular part of the diaphragm.

Frank L. Caes, $M D^{a}$

Bart Francois, $M D^{b}$

Guido J. Van Nooten ${ }^{a}$

Department of Cardiac Surgery

University Hospital

Ghent, Belgium ${ }^{a}$

Division of Cardiology

St.-Jozef Hospital

\section{REFERENCES}

Ghentbrugge, Belgium ${ }^{b}$

1. Suma H, Wanibuchi Y, Terada Y, Fukuda S, Takayama T, Furuta $\mathrm{S}$. The right gastroepiploic artery graft: clinical and angiographic midterm results in 200 patients. J THORAC Cardiovasc Surg 1993; 105:615-23.

2. Lytle BW, Cosgrove DM, Ratliff NB, Loop FD. Coronary artery bypass grafting with the right gastroepiploic artery. $\mathrm{J}$ Thorac Cardiovasc SuRg 1989;97:826-31.

3. Mills NL, Hockmuth DR, Everson CT, Robart CC. Right gastroepiploic artery used for coronary artery bypass graft- 
ing: evaluation of flow characteristics and size. J THORAC Cardiovasc Surg 1993;106:579-86.

4. Calabuig R, Moody FG. Abdominal cavity: anatomy and structural anomalies. In: Yamada T, ed. Textbook of gastroenterology. Philadelphia: JB Lippincott, 1991:2045-56.

\section{Successful correction of Ebstein's anomaly with rheumatic mitral valvular disease}

\section{To the Editor:}

Anomalies of the left side of the heart associated with Ebstein's malformation of the tricuspid valve occur occasionally. Mitral valve abnormalities that have been reported include valvular stenosis, parachute mitral valve, mitral valve prolapse, supravalvular ring, cleft mitral leaflet in association with endocardial cushion defects, coarctation of the aorta, and other defects. A combination of Ebstein's anomaly with rheumatic mitral valvular disease is still a rarity. We report here a case of rheumatic mitral valvular lesion and Ebstein's anomaly, successfully treated with tricuspid and mitral valve replacements. Successful treatment of this combination of lesions has not been reported previously to our knowledge.

An 11-year-old Kenyan boy weighing $25.5 \mathrm{~kg}$ was admitted with a history of frequent cough and palpitations of 7 years' duration. There was a history of dyspnea on exertion and paroxysmal nocturnal dyspnea since the age of 3 years, and of pain on the left side of the chest, puffiness of the face, and edema of the lower limbs since the age of $3 \frac{1}{2}$ months. Clinical examination revealed clubbing and pallor. Heart rate was 98 beats $/ \mathrm{min}$ and regular. Jugular venous pulsations were within normal limits. Blood pressure was $90 / 70 \mathrm{~mm} \mathrm{Hg}$. Apex beat was in the left sixth intercostal space, outside the midclavicular line. The first heart sound was normal; the pulmonary component of the second heart sound was loud. Auscultation findings suggested mitral stenosis with regurgitation and tricuspid regurgitation. The liver was $4 \mathrm{~cm}$ below the right costal margin and pulsatile. Results of routine blood and urine investigations were within normal limits. A chest radiograph showed significant cardiomegaly, with a narrow pedicle and normal pulmonary blood flow. There was mitralization of the left heart border. Electrocardiogram showed normal sinus rhythm, with right and left atrial enlargement. Echocardiography revealed trabeculated right ventricle with Ebstein's anomaly of the tricuspid valve. There was moderate tricuspid regurgitation. The mitral valve had a restricted opening, with doming of the leaflets associated with mitral regurgitation. Cardiac catheterization confirmed the echocardiographic findings, and the characteristic cloverleaf appearance of the tricuspid valve could be discerned (Fig. 1). The catheterization data are given in Table I. The diagnosis of Ebstein's anomaly with moderate tricuspid regurgitation and rheumatic mitral stenosis with regurgitation (Fig. 2) was established. The patient was taken in for surgical correction. Surgical exposure was through a median sternotomy. Cardiopulmonary bypass was achieved by cannulation of the aorta, superior vena cava, and inferior vena cava. The patient was cooled to $25^{\circ}$ C. Cold blood cardioplegia was used to achieve cardioplegic arrest; topical hypothermia with normal saline solution was used in addition. The tricuspid anulus was extremely large, with a perforated anterior leaflet. It was therefore decided to replace the valve with a $3 \mathrm{M}$ Starr-Edwards valve (Baxter Healthcare Corp., Edwards Division, Santa Ana, Calif.) attached with continuous 2-0 polypropylene sutures in a supracoronary position. The aneurysmal part of the atrialized right ventricular portion was also plicated. The mitral valve was excised and

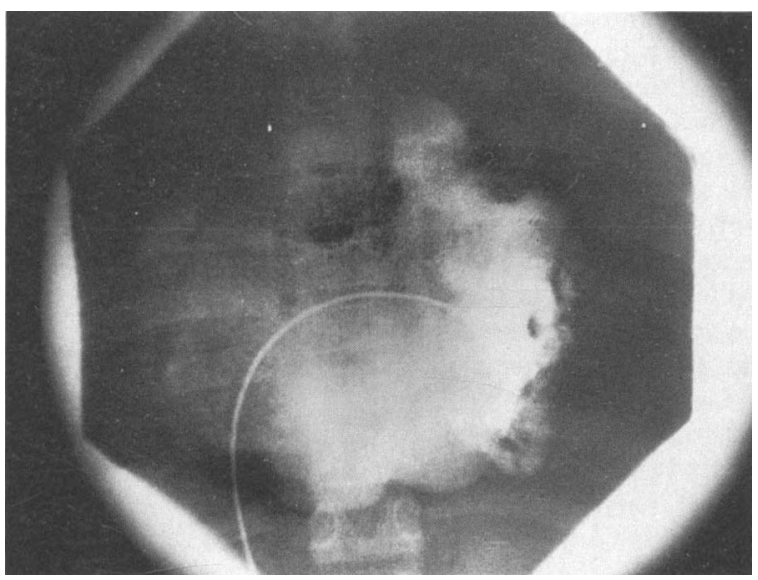

Fig. 1. Angiocardiogram shows the typical cloverleaf deformity of Ebstein's anomaly.

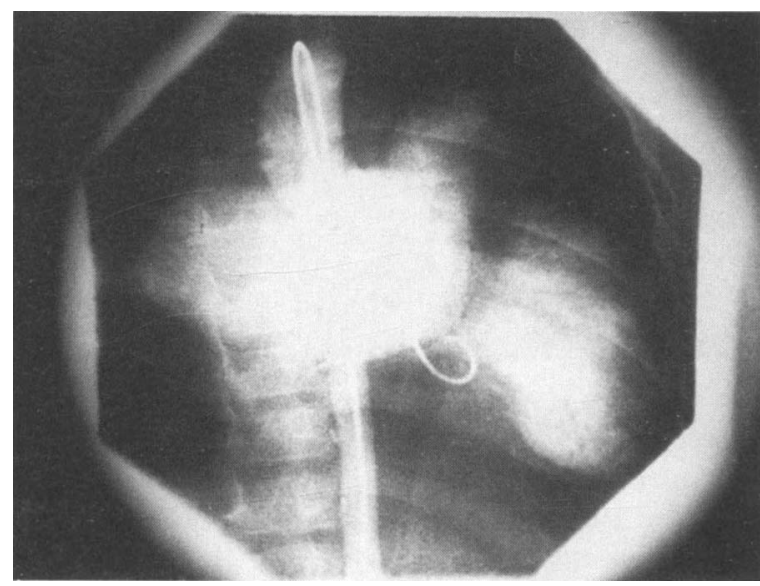

Fig. 2. Angiocardiogram shows gross mitral regurgitation.

Table I. Hemodynamic data

\begin{tabular}{lcc}
\hline & & Pressure in $\mathrm{mm} \mathrm{Hg}$ \\
\cline { 3 - 3 } \multicolumn{1}{c}{ Site } & Oxygen saturation & Syst/dias (mean) \\
\hline HSVC & 43.8 & \\
IVC & 23 & \\
MRA & 32.6 & $18 / 16(14)$ \\
MRV & 34.1 & $38 / 12$ \\
PA & 33.6 & $38 / 12(25)$ \\
LPW & & $32 / 35(25)$ \\
LV & 97 & $100 / 8$ \\
AA & 95.4 & $100 / 68(83)$ \\
FA & & $112 / 70(82)$ \\
RV-RA gradient & & 3 \\
PA-RV gradient & & 0 \\
LV-AO gradient & & 0 \\
PW-LVEDP gradient & & 17 \\
\hline
\end{tabular}

$H S V C$, High superior vena cava; $I V C$, inferior vena cava; $M R A$, mid right atrium; $M R V$, mid right ventricle; $P A$, pulmonary artery; $L P W$, left pulmonary wedge pressure; $L V$, left ventricle; $A A$, ascending aorta: $F A$, femoral artery; $R V$, right ventricle; $R A$, right atrium; $A O$, aorta; $L V E D P$, left ventricular enddiastolic pressure. 